\title{
È tempo di consuntivi
}

Monaldi Arch Chest Dis 2006; 66: 161-163.

Sono già trascorsi due anni dalla mia nomina a presidente del Gruppo di Cardiologia Riabilitativa e preventiva e prima di passare il testimone credo sia opportuno fare un bilancio del nostro operato: delle cose che abbiamo fatto e di quelle che avremmo voluto fare.

Abbiamo lavorato, insieme agli amici del direttivo, con la massima dedizione con l'obbiettivo di mantenere elevati i livelli di eccellenza già raggiunti e nel tentativo di proporre al mondo cardiologico il nostro modo di lavorare e le nostre proposte. Il contesto nel quale abbiamo agito, non è facile, lo sapevamo, dal momento che viviamo in un'epoca in cui se da un lato vi è la consapevolezza che la assenza di una vera continuità assistenziale rischia di vanificare gran parte dei risultati che si ottengono nel trattamento della fase acuta della malattia, dall'altro la cultura della prevenzione stenta ad affermarsi nel nostro, come nella maggioranza degli altri paesi.

Il filo conduttore del nostro operato è stato quello di cercare un dialogo e una collaborazione sempre più stretta con le altre società di settore cercando di rapportarci con queste nel modo più schietto possibile in modo da riuscire a capire quali fossero le nostre debolezze e nello stesso tempo di sottolineare con forza quale può essere il valore aggiunto della cardiologia riabilitativa nei vari contesti assistenziali.

\section{Che cosa abbiamo fatto}

\section{Al nostro interno}

Il consiglio direttivo si è riunito molte volte, compatibilmente con le possibilità economiche notoriamente non brillanti, almeno a detta del tesoriere che ci ha sempre ammonito sulla copertura finanziaria di ogni movimento. Come previsto tutti hanno contribuito in modo significativo a portare avanti il programma che avevamo concordato all'inizio del biennio.

Abbiamo riscritto e approvato il nuovo statuto della società in modo da uniformarlo alle necessità attuali e renderlo idoneo ad essere recepito nelle sedi istituzionali adeguate.

Abbiamo provveduto a nominare i nuovi delegati regionali ai quali è stato affidato il compito di promuovere ogni iniziativa utile per colmare lo scollamento certamente esistente tra centro e periferia.

Abbiamo aggiornato il nostro sito cercando di farne uno strumento da consultare con facilità e che possa essere utile nell'informare i soci sulle iniziative formative e culturali del gruppo e sulle problematiche emergenti.
Abbiamo nominato condirettore del giornale Carlo Vigorito certi che la sua competenza, il suo entusiasmo e la sua estrazione possano dare un contributo significativo in un ambito che ci ha creato, ad un certo momento, non pochi problemi.

Abbiamo avviato lo studio SIGARETTA nella convinzione che la abolizione del fumo sia uno degli obiettivi prioritari di un programma di prevenzione secondaria laddove è documentato che la sua sospensione dopo un evento coronarico acuto sia in grado di ridurre la mortalità in modo impressionante e soprattutto infinitamente di più di qualsiasi altro approccio anche aggressivo.

Abbiamo costruito il programma del nostro prossimo Congresso, che si terrà a Palermo e che destinerà un ruolo centrale alla definizione dei percorsi clinico assistenziali degli "attuali" pazienti cardiopatici da quelli sottoposti a rivascolarizzazione meccanica o a impianto di device elettrici a quelli cardioperati che rappresentano una "nuova" popolazione per le loro caratteristiche di età avanzata, disfunzione ventricolare sinistra grave e comorbilità. Uno spazio importante sarà riservato alla fisiologia e agli effetti dell'esercizio fisico sull'endotelio, sulla funzione cardiaca, sul sistema nervoso autonomo, sull'infiammazione con ricadute importanti sulla evoluzione dei pazienti ad alto rischio. Sono in programma simposi congiunti con l'Area Prevenzione dell'ANMCO e con il GISE e molto altro con l'obbiettivo di promuovere la massima aggregazione possibile tra $\mathrm{i}$ vari componenti del team riabilitativo: cardiologo,infermiere, fisioterapista, dietista, psicologo. Pantaleo Giannuzzi, Chairman dello studio Gospel, presenterà i risultati definitivi di un lavoro che ha impegnato per un lungo periodo la cardiologia riabilitativa nel nostro paese e che avrà certamente un impatto importante sulla pratica clinica e una notevole risonanza nella comunità cardiologia nazionale e internazionale.

Raffaele Griffo ha "inventato" la newsletter del consiglio direttivo che consentirà ai soci, anche più "distratti", di essere aggiornati sulle vita della società: è un impegno difficile da portare avanti con continuità, ma certamente molto utile

Sotto l'impulso dell'agenzia per i Servizi Sanitari Regionali, nell'ambito del programma nazionale linee guida abbiamo contribuito (Iesi, Griffo, Giannuzzi, Urbinati) alla stesura delle linee guida in cardiologia riabilitativa e preventiva che hanno una importanza fondamentale non solo sul piano scientifico ma anche dal punto di vista dei rapporti con il decisore pubblico che peraltro appare sempre più attento ai bisogni del paziente ad alto rischio e ormai 
convinto del favorevole rapporto costo-beneficio della riabilitazione e della prevenzione cardiovascolare.

\section{Nei confronti delle altre società}

Alla stregua della società europea di cardiologia che ha promosso la nascita di una unica società di prevenzione e riabilitazione e con il sostegno convinto del presidente dell'ANMCO Giuseppe Di Pasquale abbiamo avviato rapporti molto stretti con l'Area Prevenzione dell'ANMCO nel tentativo di arrivare in un tempo non lontano ad una fusione di fatto dei due gruppi. In questa fase abbiamo cercato, con successo, di portare avanti iniziative comuni in ambito formativo come testimoniato dai simposi congiunti al congresso ANMCO, al prossimo congresso della nostra società e ai corsi clinico pratici di cardiologia preventiva e riabilitativa sui percorsi assistenziali integrati e condivisi tra ospedale e territorio che abbiamo reiterato in molte città d'Italia e che sono state molto apprezzati dai nostri soci.

In considerazione della nota dicotomia esistente tra gestione della fase acuta della malattia, certamente sempre più aggressiva, più costosa, ma anche più efficace, e quella della fase cronica ancora troppo poco considerata, abbiamo promosso la I Conferenza della Cardiologia Riabilitativa e Preventiva in era interventistica che per la prima volta ha riunito intorno allo stesso tavolo i cardiologi interventisti, gli elettrofisiologi, i cardiologi che si occupano di scompenso cardiaco, i medici di medicina generale, i rappresentanti delle istituzioni (Ministero della Salute) e i cardiologi che si occupano di riabilitazione e prevenzione. La manifestazione tenutasi a Napoli nei primi mesi dell'anno grazie all'impegno di Chieffo, al contributo non condizionante di Sigma Tau e ovviamente dei membri del direttivo oltre che di Temporelli, che hanno messo a punto un programma scientifico di alto livello, è stato un passaggio che riteniamo fondamentale per conoscere le aspettative dei colleghi che si occupano del paziente acuto e mettere in evidenza le nostre proposte in termini di gestione a lungo termine del paziente cardiopatico. Io sono convinto che questa iniziativa debba essere reiterata, magari a cadenza biennale, dal momento che il confronto sereno e serrato tra le varie figure professionale e la condivisione dei percorsi clinico assistenziali più adeguati dovrebbe portare ad una accettazione più convinta di una attività che ha già dimostrato, peraltro, un rapporto costo-benefico particolarmente favorevole.

In un momento in cui il clima federativo e in particolare i rapporti tra ANMCO e SIC sono certamente molto buoni, abbiamo aderito con convinzione al consiglio federale delle società cardiologiche dal momento che siamo convinti che molti dei problemi della nostra società, come del resto quelli delle altre, possano essere affrontati con maggiori possibilità di successo tenendo unite le varie anime della cardiologia: "le società di settore che fanno parte del Consiglio Federale dovranno essere riconosciute come referenti della cardiologia italiana nei confronti della ESC e delle Istituzioni sanitarie per gli ambiti di propria competenza".
Con l'Area Scompenso Cardiaco e l'Area Management e Qualità dell'ANMCO abbiamo partecipato alla stesura del documento di consenso su "Il percorso assistenziale del paziente con scompenso cardiaco" con l'obbiettivo di definire i principi che devono ispirare i percorsi diagnostici e terapeutici del paziente con scompenso cardiaco in continuità assistenziale tra ospedale e territorio ed in stretta collaborazione con tutte le figure professionale coinvolte pur nel rispetto delle specificità locali.

Nei confronti di SINFER, associazione dei fisiatri, che rivendicano centralità e titolarità della riabilitazione cardiologica abbiamo sottolineato, che la RC nelle sue varie articolazioni organizzative, degenziale, day hospital, ambulatoriale, è, nel nostro paese, da molti anni operante nell'ambito di unità operative complesse a direzione cardiologica o in strutture semplici afferenti a UO di cardiologia. Questa integrazione garantisce, come definito dal documento della Federazione Italiana di Cardiologia, la gestione della fase postacuta, ma anche la continuità assistenziale di patologie croniche, permettendo la realizzazione di un modello gestionale appropriato nelle varie fasi della malattia. Sono ormai consolidate le evidenze che documentano come la RC non si identifica con l'esercizio fisico e come la stessa applicazione di quest'ultimo in pazienti a rischio elevato richieda comunque conoscenze fisiopatologiche e cliniche tipiche ed esclusive della cultura cardiologica. La titolarità e la responsabilità di governo della $\mathrm{RC}$ dunque non possono dunque che essere della cardiologia al di là delle improvvide richieste di specialisti nel cui percorso formativo è assente ogni contenuto cardiologico.

\section{Che cosa avremmo voluto fare}

Certamente avremmo voluto fare di più in molti ambiti: una più completa integrazione con l'area prevenzione dal momento che le malattie cardiovascolari rimangono la prima causa di morte e di disabilità nonostante i progressi della terapia farmacologica e la attenzione sempre crescente verso un atteggiamento aggressivo delle sindromi coronariche acute e d'altra parte un approccio attivo alla prevenzione si basa su una serie di evidenze consolidate; una maggiore attenzione al problema relativo alle difficoltà di rapportarci con gli altri cardiologi soprattutto quelli che gestiscono la fase acuta e quelli che operano nell'ambito della insufficienza cardiaca dal momento che la $\mathrm{R} C$ è per vocazione indirizzata alla gestione del paziente cronico e pertanto può giocare un ruolo preminente nella ottimizzazione della terapia, nella stratificazione del rischio, nella promozione dell'esercizio fisico in grado di migliorare la qualità della vita e la sopravvivenza.

E ancora non siamo riusciti ad avviare gli studi che avevamo programmato sulla efficacia della RC nei pazienti con infarto miocardio acuto e trattati con angioplastica coronarica e sulla valutazione a distanza dei pazienti operati di bypass coronarico che pure afferiscono numerosi alle nostre strutture. E stato soprattutto un problema di reperimento delle risorse economiche adeguate, ma forse un nostro maggiore impegno e una nostra maggiore convin- 
zione avrebbero potuto supplire a queste difficoltà che peraltro diventano sempre più grandi.

Anche nei confronti della periferia avremmo voluto e potuto fare di più soprattutto migliorando la comunicazione e coinvolgendo maggiormente i delegati regionali sempre in grado di fornire contributi rilevanti: Diaco, ad esempio, ha organizzato una serie di incontri formativi in regione Lombardia il cui format potrebbe essere "esportato" e implementato alla luce delle esigenze locali.

In conclusione, credo che le cose che abbiamo fatto non sono poche; sulla qualità del lavoro svolto saranno i soci a giudicare, spero con benevolenza, dal momento che abbiamo operato con il massimo impegno nonostante le difficoltà che sono obiettivamente molte. Come ricordato da Franco Chiarella all'inizio del suo mandato come presidente dell'ANMCO "l'aumento dell'età media dei cardiologi ospedalieri, la mancanza di ricambio nelle unità operative, la difficile realtà sanitaria nazionale caratterizzata dal blocco delle assunzioni e dalla provvisorietà degli incarichi hanno indotto disillusione disillusione e caduta motivazionale in senso professionale e associativo".
Quello che di buono è stato fatto si deve all'impegno degli amici del direttivo cui va un ringraziamento non formale, ma anzi affettuoso e caloroso: Francesco Fattirolli, che ha continuato ad operare con discrezione e lucidità, Raffaele Griffo cui spetterà il compito di guidare la associazione nei prossimi due anni, certamente lo farà nel migliore dei modi per le sue capacità e per la sua profonda conoscenza della società stessa, Mario Chiatto con il suo entusiasmo e la sua capacità di affrontare criticamente ogni problematica, Maurizio Ferratini con la sua esperienza maturata in tanti anni al centro De Gasperis, Margherita Vona entusiasta del suo lavoro e della mission della società, Pier Luigi Temporelli che ha fatto splendidamente il tesoriere e non solo, Carlo Vigorito per la sua competenza e la sua signorilità. Un ringraziamento infine a Carmine Chieffo che ci è sempre stato vicino anche nei momenti più difficili, alla signora M. Luisa Briolotti puntuale ed efficiente e ai delegati regionali che pur tra tante difficoltà hanno sempre cercato di operare al meglio.

Un augurio sincero di buon lavoro a tutti.

Salvatore Pirelli 